Bundesgesundheitsbl $2021 \cdot 64: 1522-1532$ https://doi.org/10.1007/s00103-021-03455-1 Eingegangen: 30 . Juni 2021

Angenommen: 22. Oktober 2021

Online publiziert: 9. November 2021

(c) Der/die Autor(en) 2021

Manfred Döpfner,', · Julia Adam ${ }^{1}$ Carolina Habbel' · Birte Schulte ${ }^{2}$. Karen Schulze-Husmann ${ }^{1} \cdot$ Michael Simons $^{3} \cdot$ Fabiola Heuer $^{4} \cdot$ Christiane Wegner $^{5}$. Stephan Bender ${ }^{2} \cdot$ TEMPO-Studiengruppe $\cdot$ B-FAST-Studiengruppe

'Ausbildungsinstitut für Kinder- und Jugendlichenpsychotherapie (AKiP), Medizinische Fakultät und Uniklinik Köln, Universität zu Köln, Köln, Deutschland; ${ }^{2}$ Klinik für Psychiatrie, Psychosomatik und Psychotherapie des Kindes- und Jugendalters, Medizinische Fakultät und Uniklinik Köln, Universität zu Köln, Köln, Deutschland; ${ }^{3}$ Klinik für Psychiatrie, Psychosomatik und Psychotherapie des Kindes- und Jugendalters, RWTH Aachen, Aachen, Deutschland; ${ }^{4}$ Klinik für Psychiatrie, Psychosomatik und Psychotherapie des Kindes- und Jugendalters, Charité, Universitätsmedizin Berlin, Berlin, Deutschland; ${ }^{5}$ Klinik für Kinder- und Jugendpsychiatrie/Psychotherapie, Universitätsmedizin Göttingen, Georg-AugustUniversität Göttingen, Göttingen, Deutschland

\title{
Die psychische Belastung von Kindern, Jugendlichen und ihren Familien während der COVID-19- Pandemie und der Zusammenhang mit emotionalen und Verhaltensauffälligkeiten
}

der Kinder und Jugendlichen [3-5] sowie 30-75\% der Bezugspersonen erhöhte Belastungen schildern [4, 6, 7]. Einige Studien zeigen erhöhte Raten von Depressionen und Angstsymptomen sowohl bei Kindern als auch bei Bezugspersonen [6, 8-10], teilweise auch erhöhte Hyperaktivität sowie vermehrte Unaufmerksamkeit und oppositionelles Verhalten $[4,11]$. In der ersten deutschlandweiten repräsentativen Studie, der COPSY-Studie [12, 13], gaben $71 \%$ der Kinder und Jugendlichen und $75 \%$ der Eltern an, dass sie sich durch die erste Welle der Pandemie belastet fühlten. Im Vergleich $\mathrm{zu}$ den Ergebnissen vor der Pandemie war die Lebensqualität der Kinder bedeutsam reduziert und die Rate der Kinder mit psychischen Auffälligkeiten verdoppelte sich annähernd von $9,9 \%$ auf $17,8 \%$.

Die Vermutung liegt nahe, dass durch die COVID-19-Pandemie psychische Belastungen und Störungen bei bereits psychisch auffälligen Kindern und Jugendlichen stärker ausgeprägt sind als bei psychisch unauffälligen. Einige Studien konnten erhöhte Raten von psychischen
Belastungen und Störungen bei Kindern mit vorbestehenden psychischen Auffälligkeiten nachweisen [11, 14], andere nicht [15-17].

Eine Erfassung von psychischen Belastungen und Störungen sowohl im Selbsturteil von Kindern und Jugendlichen als auch im Elternurteil ist deshalb besonders aufschlussreich, weil das Selbsturteil erheblich vom Urteil der Eltern differieren kann $[18,19]$. Allerdings liegt unseres Wissens bislang nur eine Studie vor, die Selbst- und Elternurteil zu Belastungen und psychischen Auffälligkeiten von Kindern und Jugendlichen mit und ohne psychische Störungen während der Coronapandemie parallel erhoben hat [20]. In dieser kanadischen Studie konnte bei rund $70 \%$ der Kinder und Jugendlichen in zumindest einem von 6 Bereichen (Depression, Angst, Reizbarkeit, Unaufmerksamkeit, Hyperaktivität, Zwanghaftigkeit) der psychischen Gesundheit eine Verschlechterung festgestellt werden, wobei die Unterschiede zwischen Kindern und Jugendlichen mit und ohne psychische Störungen gering waren. 


\begin{tabular}{|c|c|c|c|c|c|}
\hline & Gesamt & $\begin{array}{l}\text { Aachen und } \\
\text { Göttingen }\end{array}$ & Berlin & $\begin{array}{l}\text { Köln } \\
\text { AKiP }\end{array}$ & $\begin{array}{l}\text { Köln } \\
\text { KJP }\end{array}$ \\
\hline Ambulanztermin vereinbart: $n$ & 1054 & 282 & 248 & 287 & 237 \\
\hline Erstvorstellungen & 493 & 33 & 41 & 287 & 132 \\
\hline Wiedervorstellungen & 561 & 249 & 207 & 0 & 105 \\
\hline Kontraindikation: $n$ & 447 & 122 & 160 & 27 & 138 \\
\hline Indikation: $n$ & 604 & 158 & 88 & 258 & 100 \\
\hline Kein Studieninteresse/Studienabbruch & 324 & 111 & 39 & 157 & 17 \\
\hline Studieninteresse und Einwilligung & 280 & 47 & 49 & 101 & 83 \\
\hline
\end{tabular}

Neben Belastungen können die Veränderungen im Zusammenhang mit der Pandemie auch zu Entlastungen bei Kindern, Jugendlichen und ihren Familien führen [21, 22]. So kann das Aussetzen des normalen Schulbesuchs schulischen Stress verringern, die Reduktion von Gleichaltrigenkontakten kann Kinder und Jugendliche mit Kontaktproblemen zunächst entlasten. Solche Entlastungen wurden bislang zwar wesentlich seltener erhoben, Cost et al. [20] fanden jedoch bei $19-31 \%$ der Kinder und Jugendlichen in mindestens einem Bereich eine Verbesserung der psychischen Gesundheit.

Die aktuelle Studie untersucht daher im Zeitraum Herbst 2020 bis Frühjahr 2021 in einer Klinikstichprobe und einer (Vor-)Schulstichprobe (1) die Stärke der psychischen Belastung und der Veränderung psychischer Auffälligkeiten von Kindern und Jugendlichen während der Pandemie im Urteil der Eltern und der Kinder und Jugendlichen selbst (ab 11 Jahre) sowie (2) den Zusammenhang von psychischer Belastung mit zeitgleich erhobenen Verhaltens- und emotionalen Auffälligkeiten der Kinder und Jugendlichen im Selbst- und im Elternurteil sowie mit soziodemografischen Parametern.

\section{Methoden}

Die Studie wurde im Rahmen des Netzwerkes Universitätsmedizin $\left(\mathrm{NUM}^{1}\right)$, das vom Bundesministerium für Bildung und Forschung (BMBF) gefördert wird, in 2 multizentrischen Projekten durch-

1 https://www.netzwerk-universitaetsmedizin. de/. geführt. Die Klinikstichprobe wurde im Rahmen der TEMPO-Studie (Telemedizinische Psychiatrie und Psychotherapie für Kinder und Jugendliche während der COVID-19-Pandemie) (registriert im Deutschen Register Klinischer Studien, DRKS-ID: 00023525) erhoben. Die (Vor-)Schulstichprobe wurde im Rahmen des Projektes B-FAST Schulen und Kitas (Bundesweites Forschungsnetz Angewandte Surveillance und Testung) gewonnen (DRKS-ID: RKS00023911).

Die Daten wurden anhand des Datenerfassungstools REDCap (Research Electronic Data Capture) erhoben. Die Teilnehmenden füllten dabei Fragebögen über einen per E-Mail an sie versandten Link selbst aus. Es wurden deskriptive Statistiken berechnet sowie Korrelations- und Varianzanalysen durchgeführt. T-Tests wurden zum Vergleich von Mittelwertunterschieden zwischen Beurteilungsperspektiven (und Erhebungszeiträumen) innerhalb einer Stichprobe durchgeführt. Vergleiche zwischen den Stichproben (Feldstichprobe vs. Klinikstichprobe) erfolgten ausschließlich deskriptiv.

\section{Stichproben und Rekrutierung}

Die Klinikstichprobe umfasst $n=280 \mathrm{~Pa}$ tient:innen zwischen 4 und 17 Jahren, die im Rahmen der Routineversorgung an kinder- und jugendpsychiatrischen Institutsambulanzen von 4 Universitätskliniken (Aachen: $n=7$, Charité Berlin: $n=49$, Göttingen: $n=40$, Köln: $n=83$ ) und einer Psychotherapieambulanz für Kinder und Jugendliche (AKiP Uniklinik Köln: $n=101$ ) im Zeitraum vom 01.12.2020 bis 31.03.2021 (Pandemiewelle 2 bis erstes
Drittel Pandemiewelle 3) vorgestellt wurden.

In die Studie wurden konsekutiv angemeldete, wiedervorgestellte bzw. weiterbehandelte Patient:innen (Alterskriterium: 4 bis 17 Jahre) eingeschlossen. Voraussetzung hierfür war die schriftliche Einwilligung der Sorgeberechtigten und Patient:innen ab 8 Jahren in die Studienteilnahme. Da das Hauptziel dieser Studie die Untersuchung der Umsetzung und Durchführung von Videosprechstunden war, wurden Patient:innen ausgeschlossen, bei denen (nach klinischem Urteil) eine Kontraindikation für eine Videosprechstunde vorlag, wie eine akute psychiatrische Notfallsituation, eine geistige Behinderung oder eine akute körperliche Symptomatik, die unmittelbar eine somatische Diagnostik bzw. Behandlung erforderte. Zudem wurden Familien mit mangelnden Deutschkenntnissen ausgeschlossen. Die Diagnosen der Klinikstichprobe basieren ebenfalls auf einem klinischen Urteil. Eine Übersicht über die Rekrutierung an den einzelnen Standorten findet sich in $\bullet$ Tab. 1.

Die (Vor-)Schulstichprobe umfasst $n=1958$ Kinder und Jugendliche zwischen 4 und 19 Jahren, die in Kitas oder Schulen an 5 Standorten (Düsseldorf, Heidelberg, Homburg, Köln, München) im Zeitraum vom 09.11.2020 bis zum 18.04.2021 (Anfang Pandemiewelle 2 bis Mitte Pandemiewelle 3) befragt wurden. Die teilnehmenden Einrichtungen wurden in Absprache mit den Gesundheits- und Schulämtern sowie weiteren beteiligten Institutionen ausgewählt. Die Auswahl der Einrichtungen zielte darauf ab, Regionen mit verschiedenen Bevölkerungsdichten und sozialen Kontexten abzubilden. Insgesamt waren 18 Einrichtungen (2 Kitas, 8 Grundschulen, 8 weiterführende Schulen) beteiligt. Eine Teilnahme am Projekt konnte insgesamt rund 5000 Kindern bzw. ihren Eltern angeboten werden. Eine schriftliche Einwilligung der Eltern und Schüler:innen ab 8 Jahren war Voraussetzung für eine Studienteilnahme.

\section{Messinstrumente}

Zur Erfassung der psychischen Belastung der Kinder bzw. Jugendlichen und 
Bundesgesundheitsbl $2021 \cdot 64: 1522-1532$ https://doi.org/10.1007/s00103-021-03455-1

(c) Der/die Autor(en) 2021

M. Döpfner · J. Adam · C. Habbel · B. Schulte · K. Schulze-Husmann · M. Simons · F. Heuer · C. Wegner · S. Bender · TEMPO-Studiengruppe $\cdot$ B-FASTStudiengruppe

\section{Die psychische Belastung von Kindern, Jugendlichen und ihren Familien während der COVID-19- Pandemie und der Zusammenhang mit emotionalen und Verhaltensauffälligkeiten}

\section{Zusammenfassung}

Hintergrund und Ziel. Die im Rahmen der COVID-19-Pandemie erlassenen Maßnahmen zum Infektionsschutz führten zu tiefgreifenden Einschränkungen und Veränderungen im sozialen, (vor-)schulischen, familiären und Freizeitbereich. Die vorliegende Studie untersucht das Ausmaß an psychischer Belastung von Kindern, Jugendlichen und ihren Familien während der COVID-19-

Pandemie. Mögliche Einflussfaktoren sollen identifiziert werden.

Material und Methoden. Die Untersuchungen erfolgten zwischen Herbst 2020 und Frühjahr 2021 in einer klinischen Inanspruchnahmestichprobe ( $n=280$ Patient:innen zwischen 4-17 Jahren) und einer Feldstichprobe ( $n=1958$ Kinder und Jugendliche zwischen
4-19 Jahren, über Schulen und vorschulische Einrichtungen rekrutiert). Dabei wurden Urteile der Eltern sowie Selbsturteile der Kinder und Jugendlichen mittels Fragebögen erfasst.

Ergebnisse. Die psychische Belastung der Kinder und Jugendlichen im Zusammenhang mit der Pandemie wird über beide Beurteilungsperspektiven und Stichproben hinweg als leicht bis moderat erhöht eingeschätzt. Rund $60-70 \%$ der Eltern- und Selbsturteile beschreiben eine Zunahme dieser Belastung, während Entlastungen von bis zu $12 \%$ sowohl im Eltern- als auch im Selbsturteil angegeben werden. Beim Vergleich der beiden Stichproben zeigt sich eine leicht höhere Belastung der Kinder und Jugendlichen nur im Selbsturteil der Klinikstichprobe. Die untersuchten soziodemografischen Faktoren haben keinen Einfluss auf die Belastung. Allerdings zeigen sich in beiden Stichproben leichte bis moderate Zusammenhänge zwischen der subjektiv erlebten Verschlechterung der familiären und sozialen Situation und einem erhöhten Belastungserleben.

Diskussion. Während einer Pandemie sollten gezielte Interventionen für belastete Subgruppen angeboten werden. Universelle Interventionen sind nicht indiziert.

Schlüsselwörter

COVID-19 · Kinder und Jugendliche · Psychische Belastung · Verhaltensauffälligkeiten . Emotionale Auffälligkeiten

\section{The mental burden of children, adolescents, and their families during the COVID-19 pandemic and associations with emotional and behavioral problems}

\section{Abstract}

Background and objective. The infection protection measures adopted as part of the COVID-19 pandemic led to profound restrictions and changes in the social, (pre-) school, family, and leisure areas. The objective of the current study was to examine the mental burden of children and adolescents and their families during the COVID-19 pandemic. Furthermore, this study aimed to identify possible factors that influence the mental burden.

Materials and methods. The examinations were carried out between autumn 2020 and spring 2021 in a clinical sample $(n=280$ patients aged $4-17$ years) and a community sample ( $n=1958$ children and adolescents aged 4-19 years recruited via schools and preschools). Ratings of parents as well as children and adolescents via questionnaires were assessed.

Results. Mental burden due to the corona pandemic was assessed as slightly to moderately increased across both rating perspectives and both samples. Overall, around 60 to $70 \%$ of the parents, children, and adolescents describe an increase in mental burden; in contrast, up to $12 \%$ of parents as well as children and adolescents describe relief. When comparing both samples, a slightly higher burden on children and adolescents can only be seen in the self-assessment of the clinical sample. None of the socio-demographic factors analyzed influences the mental burden statistically significant. However, low to moderate correlations between the subjectively experienced deterioration in the family and social situation and an increased level of stress is found.

Discussion. Targeted interventions for exposed subgroups should be offered during a pandemic. Universal interventions are not indicated.

\section{Keywords}

COVID-19 Children and adolescents · Mental burden · Behavior problems · Emotional problems ihrer Familien durch die COVID-19Pandemie in der Klinikstichprobe und der (Vor-)Schulstichprobe wurde eine gekürzte Version des Corona-Belastungsbogens (CBB; [23]; s. Onlinematerial 1) im Elternurteil (CBB-E; 11 Items) und im Selbsturteil (CBB-KJ; ab 11 Jahren; 7 Items) eingesetzt. Im Elternurteil wurden Veränderungen im Verhalten des Kindes oder Jugendlichen sowie $\mathrm{Be}$ - und Entlastungen in seinen Be- ziehungen und der Betreuungs-, der familiären, schulischen, beruflichen $\mathrm{Si}$ tuation erfasst wie auch vorhandene Freizeitmöglichkeiten. Die Gesamtbeurteilungen der Veränderungen für das Kind und den Elternteil selbst wurden anhand von fünfstufigen Likert-Skalen vorgenommen $(-2=$,viel besser“, $-1=$ „,etwas besser“, 0 = keine Änderung, $1=$ „etwas schlechter“, 2 = „viel schlechter"). Im Selbsturteil wurden analog zum
Elternurteil die gleichen Items erhoben bis auf die Beurteilung der Betreuung des Kindes, der familiären Situation insgesamt, der beruflichen Situation der Eltern und der Veränderungen für das Elternteil insgesamt. Es wurde jeweils ein Gesamtbelastungsindex über alle Items (Kennwert = Summe der Itemwerte/Anzahl der Items) gebildet, wobei ein höherer Wert eine höhere Belastung durch die Coronapandemie bedeutet. 
Für direkte Vergleiche zwischen CBB-E und CBB-KJ auf Skalenebene wurde ein CBB-Cross-Informant-Index mit den jeweils im Eltern- und Selbsturteil korrespondierenden Items gebildet (7 Items). Um die subjektiv erlebten Veränderungen der familiären Rahmenbedingungen abzubilden, wurde zudem ein Index (CBB-E-Rahmenbedingungen) über die Items zu Veränderungen der Betreuungs-, beruflichen und familiären Situation sowie über die Veränderungen für die Bezugsperson selbst gebildet. Die internen Konsistenzen (Cronbachs Alpha) sind bis auf den Index CBBE-Rahmenbedingungen (Klinikstichprobe: $\alpha=0,59$; (Vor-)Schulstichprobe: $\alpha=0,63$ ) für die Gesamtindizes [und den Cross-Informant-Index] sowohl für die Klinik- als auch die (Vor-)Schulstichprobe als ausreichend bis gut $\mathrm{zu}$ bewerten (CBB-E: Klinikstichprobe: $\alpha=0,81 \quad[0,79]$, (Vor-)Schulstichprobe: $\alpha=0,80 \quad[0,75]$; CBB-KJ: Klinikstichprobe: $\alpha=0,67$, (Vor-)Schulstichprobe: $\alpha=0,66)$.

Zur Erfassung von emotionalen und Verhaltensauffälligkeiten der Kinder und Jugendlichen wurden in der Klinikstichprobe der Elternfragebogen über das Verhalten von Kindern und Jugendlichen (Child Behavior Checklist/6-18 R [CBCL] [24]) und der Fragebogen für Jugendliche (Youth Self Report/11-18 R [YSR]; ab 11 Jahren [24]) eingesetzt.

In der (Vor-)Schulstichprobe wurde zur Erfassung von emotionalen und Verhaltensauffälligkeiten der Kinder und Jugendlichen die deutschsprachige Version des Strengths and Difficulties Questionnaire (SDQ [25]) im Elternurteil (SDQE) und im Selbsturteil der Kinder und Jugendlichen ab 11 Jahren (SDQ-S) eingesetzt (s. auch [26, 27]).

Zur Beurteilung der Funktionsbeeinträchtigungen und des Leidensdrucks der Kinder und Jugendlichen wurden in der Klinikstichprobe jeweils 5 Items zur Erfassung der Beeinträchtigungen der Beziehungen, der schulischen Leistungsfähigkeit und des subjektiven Leidensdrucks (Skala: $0=$ gar nicht bis 3 =besonders) aus dem Fremd- bzw. Selbstbeurteilungsbogen (ab 11 Jahren) zum Screening psychischer Störungen (FBB-SCREEN/SBBSCREEN) aus dem Diagnostik-System für psychische Störungen nach ICD-10 und DSM-5 für Kinder und Jugendliche (DISYPS-III [28]) eingesetzt.

Im klinischen Urteil erfolgte die Globalbeurteilung der psychosozialen Anpassung mithilfe der Achse 6 des Multiaxialen Klassifikationsschemas (MAS [29]) anhand einer 9-stufigen Skala $(0=$ „Hervorragende oder gute soziale Anpassung auf allen Gebieten“ bis $8=$ „Braucht ständige Betreuung [24Stunden-Versorgung]“). Mit dem Ziel einer einfacheren Interpretierbarkeit wurde die Skala für die vorliegenden Berechnungen recodiert $(0=$ „Braucht ständige Betreuung [24-Stunden-Versorgung]“ bis $8=$ „Hervorragende oder gute soziale Anpassung auf allen Gebieten“), sodass ein höherer Wert mit einer besseren psychosozialen Anpassung einhergeht.

Der sozioökonomische Status in der Klinikstichprobe wurde anhand des Berufes/der Ausbildung der Eltern erfasst, erhoben über eine Kurzfassung der Basisdokumentation [30]. Die daraus gebildete Skala zum sozioökonomischen Status reichte von (1) ungelernte Arbeiter:innen oder angelernte Berufe bis (5) leitende Angestellte, Beamt:innen im höheren Dienst oder Akademiker:innen, freie Berufe, größere Unternehmer:innen.

Weitere Informationen zu den Messinstrumenten sind dem Onlinematerial 2 zu entnehmen.

\section{Ergebnisse}

\section{Stichprobenmerkmale}

Die Merkmale der Klinikstichprobe insgesamt $(n=280)$ und der einzelnen Standorte sind - Tab. $2 \mathrm{zu}$ entnehmen (Aachen und Göttingen wurden zusammengefasst, da am Standort Aachen lediglich $n=7$ Patient:innen eingeschlossen wurden). Über alle Standorte hinweg waren die Patient:innen im Mittel 11,75 Jahre alt $(S D=3,73)$, rund $55 \%$ waren Jungen. In jeweils etwa einem Drittel der Fälle wurden ausschließlich externale Störungen (Aufmerksamkeitsdefizit-, Hyperaktivitätsstörung (ADHS), Störung des Sozialverhaltens) bzw. internale (Angst, Depression) oder gemischte/andere Störungen diagnostiziert. Die Ergebnisse von vergleichenden Analysen zwischen den Standorten finden sich im Onlinematerial 3.

In - Tab. 3 sind die Merkmale der (Vor-)Schulstichprobe ( $n=1024$ Kinderund Jugendlichenurteile; $n=1606$ Elternurteile; $n=672$ beide Urteile) dargestellt. Die im Elternurteil untersuchten Kinder waren im Mittel 10,86 Jahre alt $(S D=3,23)$, rund $45 \%$ waren Jungen, $58 \%$ besuchten eine weiterführende Schule. Die Schüler:innen, deren Selbsturteile vorlagen, waren im Mittel 13,87 Jahre alt $(S D=2,11)$, knapp $40 \%$ waren Jungen. Die Ergebnisse von vergleichenden Analysen zwischen den Substichproben (sowie Erhebungszeiträumen) finden sich im Onlinematerial 3.

\section{Stärke der psychischen Be- und Entlastungen}

Die Ausprägung der Be- und Entlastungen durch die COVID-19-Pandemie auf Itemebene in der Klinikstichprobe sowohl nach Angaben der Bezugspersonen ( $n=254,81,5 \%$ Mütter, $15,4 \%$ Väter, 3,1\% andere) als auch im Patient:innenurteil ab 11 Jahren $(n=141)$ zeigt • Tab. 4.

Die Einschätzungen der Eltern ergeben, dass die Pandemie insgesamt bei fast $24 \%$ der Patient:innen zu sehr starken und bei weiteren $48 \%$ zu leichteren negativen Veränderungen geführt hat (Item 10). Insgesamt trat also bei rund $72 \%$ eine vermehrte Belastung auf, während für rund $16 \%$ der Patient:innen keine Veränderungen und für weitere knapp $12 \%$ leichte oder sogar starke Verbesserungen, d.h. Entlastungen, angegeben wurden.

Insbesondere die Freizeit- und Beschäftigungsmöglichkeiten der Patient: innen (Item 5) haben sich nach Angabe der Bezugspersonen durch die Pandemie verschlechtert: Etwa $24 \%$ der Bezugspersonen berichteten eine leichte und fast $61 \%$ eine starke Verschlechterung der Freizeit- und Beschäftigungsmöglichkeiten ihrer Kinder. Die Beziehungen des Kindes zu anderen Familienmitgliedern (Item 1) haben sich laut der Bezugspersonen hingegen bei $52 \%$ der Patient:innen nicht verändert, bei rund $24 \%$ leicht oder stark verbessert und bei ebenfalls $24 \%$ 


\begin{tabular}{|c|c|c|c|c|c|}
\hline & Gesamt & $\begin{array}{l}\text { Aachen und } \\
\text { Göttingen }\end{array}$ & Berlin & AKiP Köln & KJP Köln \\
\hline$n$ & 280 & 47 & 49 & 101 & 83 \\
\hline Alter in Jahren: M (SD) & $11,75(3,73)$ & $13,18(3,68)$ & $10,72(3,66)$ & $11,08(3,69)$ & $12,37(3,56)$ \\
\hline Männlich: n (\%) & $153(54,6)$ & $24(51,1)$ & $29(59,2)$ & $56(55,4)$ & $44(53,0)$ \\
\hline \multicolumn{6}{|l|}{ Wohnsituation:n (\%) } \\
\hline Beide Eltern & $222(82,8)$ & $37(86,0)$ & $34(73,9)$ & $84(85,7)$ & $67(82,7)$ \\
\hline Alleinerziehend & $41(15,3)$ & $5(11,6)$ & $11(23,9)$ & $13(13,3)$ & $12(14,8)$ \\
\hline Andere & $5(1,9)$ & $1(2,3)$ & $1(2,2)$ & $1(1,0)$ & $2(2,5)$ \\
\hline \multicolumn{6}{|l|}{ Sozioökonomischer Status der Familie (nach Berufsgruppe): $n$ (\%) } \\
\hline Ungelernte Arbeiter:innen oder angelernte Berufe & $21(8,2)$ & $5(14,7)$ & $6(13,3)$ & $6(6,2)$ & $4(5,1)$ \\
\hline $\begin{array}{l}\text { Facharbeiter:innen, Handwerker:innen, Angestellte oder Beamt:innen im } \\
\text { einfachen Dienst oder kleinste Selbstständige, ambulantes Gewerbe }\end{array}$ & $53(20,8)$ & $10(29,4)$ & $7(15,6)$ & $19(19,8)$ & $17(21,3)$ \\
\hline $\begin{array}{l}\text { Mittlere Angestellte, Beamt:innen im mittleren Dienst oder kleine selbst- } \\
\text { ständige Gewerbetreibende }\end{array}$ & $79(31,0)$ & $9(26,5)$ & $14(31,1)$ & $33(34,4)$ & $23(28,7)$ \\
\hline $\begin{array}{l}\text { Höher qualifizierte Angestellte, Beamt:innen im gehobenen Dienst oder } \\
\text { Selbstständige mit mittleren Geschäften, Betrieben }\end{array}$ & $62(24,3)$ & $7(20,6)$ & $15(33,3)$ & $23(24,0)$ & $17(21,3)$ \\
\hline $\begin{array}{l}\text { Leitende Angestellte, Beamt:innen im höheren Dienst oder Akademi- } \\
\text { ker:innen, freie Berufe oder große Unternehmer:innen }\end{array}$ & $40(15,7)$ & $3(8,8)$ & $3(6,7)$ & $15(15,6)$ & $19(23,8)$ \\
\hline Psychosoziales Funktionsniveau ${ }^{a}:$ M (SD) & $5,26(1,25)$ & $5,05(1,19)$ & $4,73(1,64)$ & $5,29(1,07)$ & $5,66(1,11)$ \\
\hline Psychische Störungen in der Familie: $n$ (\%) & $133(57,8)$ & $17(50,0)$ & $27(65,9)$ & $47(58,8)$ & $42(56,0)$ \\
\hline Somatische Erkrankungen in der Familie: $n$ (\%) & $90(43,3)$ & $10(45,5)$ & $15(39,5)$ & $44(57,9)$ & $21(29,2)$ \\
\hline \multicolumn{6}{|l|}{ Diagnosen: $n(\%)$} \\
\hline Externale Störungen & $103(36,8)$ & $22(46,8)$ & $26(53,1)$ & $19(18,8)$ & $36(43,4)$ \\
\hline Internale Störungen & $85(30,4)$ & $11(23,4)$ & $7(14,3)$ & $46(45,5)$ & $21(25,3)$ \\
\hline Gemischte Störungen & $26(9,3)$ & $7(14,9)$ & $3(6,1)$ & $13(12,9)$ & $3(3,6)$ \\
\hline Andere Störungen & $66(23,6)$ & $7(14,9)$ & $13(26,5)$ & $23(22,8)$ & $23(27,7)$ \\
\hline \multicolumn{6}{|l|}{ Anzahl Diagnosen: $n(\%)$} \\
\hline 1 Diagnose & $145(51,8)$ & $21(44,7)$ & $18(36,7)$ & $62(61,4)$ & $44(53,0)$ \\
\hline 2 Diagnosen & $99(35,4)$ & $15(31,9)$ & $22(44,9)$ & $34(33,7)$ & $28(33,7)$ \\
\hline 3 Diagnosen & $36(12,9)$ & $11(23,4)$ & $9(18,4)$ & $5(5,0)$ & $11(13,3)$ \\
\hline
\end{tabular}

leicht oder stark verschlechtert. Für die Bezugspersonen selbst verschlechterte sich die Gesamtsituation nach eigener Einschätzung im Verlauf der Pandemie noch etwas stärker als bei ihren Kindern (ca. $81 \%$, Item 11).

Vergleichbar zu den Eltern gaben auch rund $19 \%$ der Kinder und Jugendlichen an, dass es ihnen durch die Coronapandemie sehr viel schlechter, und fast $47 \%$, dass es ihnen ein wenig schlechter gehe (Item 10). Auch die Kinder und Jugendlichen schätzten besonders die Freizeit- und Beschäftigungsmöglichkeiten als eher negativ verändert ein.

Gereiztheit, Unruhe und Unausgeglichenheit der Kinder oder Jugendlichen (Item 6) nahmen nach dem Eltern- und Selbsturteil in rund $14-16 \%$ der Fälle stark zu, wobei leichte Verschlechterungen noch deutlich häufiger berichtet wurden. Allerdings gibt es auch Fälle, bei denen sich diese Probleme während der Pandemie verbesserten. Ähnlich sieht es bei Ängstlichkeit, Unsicherheit und Traurigkeit (Item 7) aus, die sich bei etwa 11-19\% der Fälle nach dem Eltern- bzw. Selbsturteil stark verschlechterten. Verbesserungen traten hier laut Elternurteil aber deutlich seltener auf.

In der Substichprobe, in der die CBBBeurteilungen sowohl von Eltern als auch von Patient:innen vorliegen (Onlinematerial 4), gibt es keinen signifikanten Unterschied im Eltern- $(M=0,65$, $S D=0,56)$ und Selbsturteil $(M=0,61$, $S D=0,54)$. Die beiden korrespondieren- den Beurteilungen korrelieren zu $r=0,53$ $(p<0,001)$.

Die Ausprägungen der Be- und Entlastungen im Zusammenhang mit der Coronapandemie nach Angaben der Bezugspersonen ( $n=1606,78,7 \%$ Mütter, $20,7 \%$ Väter, 0,6\% andere) und der Kinder und Jugendlichen ab 11 Jahren $(n=1024)$ in der (Vor-)Schulstichprobe zeigt -Tab. 5. Im Vergleich zur Klinikstichprobe (-Tab.4) haben sich laut der Eltern für Kinder und Jugendliche im Verlauf der Pandemie insgesamt etwas seltener, bei knapp $17 \%$ (Vergleich Klinikstichprobe: knapp 24\%) sehr starke und bei weiteren gut $61 \%$ leichtere negative Veränderungen ergeben. Insgesamt führte die Pandemie also bei rund $78 \%$ (Klinikstichprobe: rund $72 \%$ ) 


\begin{tabular}{|c|c|c|c|}
\hline & Schüler:innen & $\begin{array}{l}\text { Eltern/Sorge- } \\
\text { berechtigte }\end{array}$ & $\begin{array}{l}\text { Gemeinsam } \\
\text { beurteilt }\end{array}$ \\
\hline$n$ & 1024 & 1606 & 672 \\
\hline Alter in Jahren: $M(S D)$ & $13,87(2,11)$ & $10,86(3,23)$ & $13,50(1,96)$ \\
\hline Altersspanne in Jahren & $11-19$ & $4-18$ & $11-18$ \\
\hline Männlich:n (\%) & $406(39,6)$ & $718(44,7)$ & $279(41,5)$ \\
\hline \multicolumn{4}{|l|}{ Einrichtungen: $n$ (\%) } \\
\hline Kita & - & $17(1,1)$ & - \\
\hline Grundstufe & - & $652(40,6)$ & - \\
\hline Weiterführende Schule & $1024(100)$ & $937(58,3)$ & $672(100)$ \\
\hline \multicolumn{4}{|l|}{ Schulstufe: $n(\%)^{a}$} \\
\hline Grundstufe (Klasse 1-4) & - & $652(42,9)$ & - \\
\hline Unterstufe (Klasse 5-6) & $249(24,3)$ & $306(20,1)$ & $184(27,4)$ \\
\hline Mittelstufe (Klasse 7-9) & $423(41,3)$ & $375(24,7)$ & $316(47,0)$ \\
\hline Oberstufe (Klasse 10-12) & $352(34,4)$ & $187(12,3)$ & $172(25,6)$ \\
\hline \multicolumn{4}{|c|}{ Fälle pro Erhebungszeitraum: $n$ (\%) } \\
\hline $11 / 2020-12 / 2020$ & $592(57,8)$ & $1160(72,2)$ & - \\
\hline $02 / 2021-04 / 2021$ & $432(42,2)$ & $446(27,8)$ & - \\
\hline
\end{tabular}

zu vermehrter Belastung, während der Zustand bei etwa $22 \%$ laut Elternurteil unverändert war. In den Mittelwerten unterscheiden sich beide Stichproben auch nominell kaum.

Ähnlich wie in der Klinikstichprobe haben sich insbesondere die Freizeit- und Beschäftigungsmöglichkeiten der Kinder nach Angabe der Bezugspersonen durch die COVID-19-Pandemie verschlechtert. Die Beziehungen der Kinder und Jugendlichen zu anderen Familienmitgliedern waren hingegen nach Einschätzung der Bezugsperson bei rund $69 \%$ unverändert, bei rund $17 \%$ verbessert und bei ca. $14 \%$ verschlechtert. Im Verlaufe der Pandemie verschlechterte sich die Gesamtsituation für die Bezugspersonen selbst noch etwas stärker als für deren Kinder (84\%).

Im Vergleich zu den Eltern gaben nur rund $8 \%$ der Kinder und Jugendlichen an, dass es ihnen durch die Coronapandemie sehr viel schlechter, und fast $54 \%$, dass es ihnen ein wenig schlechter gehe. Auch die Kinder und Jugendlichen schätzten besonders die Freizeit- und Beschäftigungsmöglichkeiten durch die Pandemie als negativ verändert ein. Im Unterschied zur Klinikstichprobe beschrieben in der (Vor-)Schulstichprobe rund $45 \%$ der Eltern keine Veränderungen hinsichtlich
Psychische Belastung und soziodemografische Parameter sowie Veränderungen im Umfeld

Zwischen soziodemografischen Merkmalen der Familien und der Gesamtbelastung durch die Pandemie zeigt sich in der Klinikstichprobe kein signifikanter Zusammenhang: Weder zum Alter der Patient:innen noch zu ihrem Geschlecht, zum sozioökonomischen Status der Familie, zur Familienzusammensetzung („getrennt“ versus „zusammenlebend“) oder zum Vorhandensein psychischer oder körperlicher Erkrankungen bei weiteren Familienmitgliedern konnte ein statistisch signifikanter Zusammenhang mit dem Gesamtbelastungsindex im Eltern- oder im Selbsturteil gefunden werden. Auch in der (Vor-)Schulstichprobe zeigen sich kaum bedeutsame Zusammenhänge zwischen soziodemografischen Merkmalen und der Gesamtbelastung durch die COVID-19-Pandemie. Lediglich zwischen Alter und dem Gesamtbelastungsindex im Selbsturteil kann ein schwacher, aber signifikanter Zusammenhang $(r=0,13, p<0,01)$ gefunden werden. Dahingegen korrelieren die subjektiv erlebten Veränderungen der familiären Rahmenbedingungen (CBB-E-Rahmenbedingungen) in beiden Stichproben statistisch signifikant mit den angegebenen Belastungen im Eltern-(CBB-Cross-Informant-Index) und Selbsturteil. Es konnte dementsprechend eine Assoziation zwischen ungünstigen Veränderungen familiärer Rahmenbedingungen durch die Pandemie und stärkeren von den Eltern (Klinikstichprobe: $r=0,54, p<0,001$; (Vor-)Schulstichprobe: $r=0,57, p<0,001)$ und Kindern bzw. Jugendlichen beurteilten Belastungen (Klinikstichprobe: $r=0,26, p<0,005$; (Vor-)Schulstichprobe: $r=0,20, p<0,001$ ) gefunden werden.

\section{Psychische Belastung und klinische Diagnosen sowie emotionale und Verhaltensauffälligkeiten}

In der Klinikstichprobe unterscheiden sich die einzelnen Diagnosegruppen (internale Störungen, externale Störungen, gemischte Störungen, andere Störungen) hinsichtlich der Belastung 
Tab. 4 Stärke von Belastungen und Entlastungen durch die Coronapandemie in der Klinikstichprobe im Elternurteil $(n=254)$ und im Selbsturteil (ab 11 Jahren; $n=141$ ), Angaben auf Itemebene

\begin{tabular}{|c|c|c|c|c|c|c|c|c|}
\hline & \multirow[t]{2}{*}{ Urteil } & \multirow[t]{2}{*}{$M$} & \multirow[t]{2}{*}{$S D$} & \multicolumn{5}{|l|}{$n(\%)$} \\
\hline & & & & $\begin{array}{l}\text { Viel } \\
\text { besser }(-2)\end{array}$ & $\begin{array}{l}\text { Etwas } \\
\text { besser }(-1)\end{array}$ & $\begin{array}{l}\text { Unverän- } \\
\text { dert (0) }\end{array}$ & $\begin{array}{l}\text { Etwas schlech- } \\
\text { ter (1) }\end{array}$ & $\begin{array}{l}\text { Viel schlech- } \\
\text { ter (2) }\end{array}$ \\
\hline \multirow{2}{*}{$\begin{array}{l}01 \text { Beziehungen Kind zu } \\
\text { Familienmitgliedern }\end{array}$} & $E$ & 0,00 & 0,87 & $11(4,3)$ & $50(19,7)$ & $132(52,0)$ & $49(19,3)$ & $12(4,7)$ \\
\hline & $\mathrm{K}$ & 0,14 & 0,87 & $3(2,1)$ & $22(15,6)$ & $70(49,6)$ & $44(31,2)$ & $2(1,4)$ \\
\hline \multirow{2}{*}{$\begin{array}{l}02 \text { Beziehungen Kind zu } \\
\text { Freund:innen }\end{array}$} & $E$ & 0,60 & 0,80 & $1(0,4)$ & $10(3,9)$ & $115(45,3)$ & $91(35,8)$ & $37(14,6)$ \\
\hline & $\mathrm{K}$ & 0,44 & 0,91 & $5(3,5)$ & $11(7,8)$ & $57(40,4)$ & $53(37,6)$ & $15(10,6)$ \\
\hline \multirow{2}{*}{$\begin{array}{l}\text { O3 Belastungen durch } \\
\text { Schule/Lernen }\end{array}$} & $E$ & 0,42 & 1,08 & $13(5,1)$ & $34(13,4)$ & $84(33,1)$ & $79(31,1)$ & $44(17,3)$ \\
\hline & K & 0,58 & 1,05 & $3(2,1)$ & $22(15,6)$ & $35(24,8)$ & $52(36,9)$ & $29(20,6)$ \\
\hline \multirow[t]{2}{*}{04 Betreuung des Kindes } & $\mathrm{E}$ & 0,40 & 0,90 & $5(2,0)$ & $28(11,0)$ & $112(44,1)$ & $79(31,1)$ & $30(11,8)$ \\
\hline & K & - & - & - & - & - & - & - \\
\hline \multirow{2}{*}{$\begin{array}{l}05 \text { Freizeit/Beschäftigungs- } \\
\text { möglichkeit }\end{array}$} & $\mathrm{E}$ & 1,42 & 0,84 & $3(1,2)$ & $3(1,2)$ & $32(12,6)$ & $62(24,4)$ & $154(60,6)$ \\
\hline & K & 1,16 & 0,93 & $1(0,7)$ & $5(3,5)$ & $30(21,3)$ & $39(27,7)$ & $66(46,8)$ \\
\hline \multirow{2}{*}{$\begin{array}{l}06 \text { Gereiztheit/Unruhe/ } \\
\text { Unausgeglichenheit }\end{array}$} & $E$ & 0,50 & 0,99 & $5(2,0)$ & $37(14,6)$ & $79(31,1)$ & $93(36,6)$ & $40(15,7)$ \\
\hline & $\mathrm{K}$ & 0,65 & 0,89 & $4(2,8)$ & $8(5,7)$ & $41(29,1)$ & $69(48,9)$ & $19(13,5)$ \\
\hline \multirow{2}{*}{$\begin{array}{l}07 \text { Ängstlichkeit/Unsicherheit/ } \\
\text { Traurigkeit }\end{array}$} & $\mathrm{E}$ & 0,41 & 0,82 & $4(1,6)$ & $15(5,9)$ & $134(52,8)$ & $74(29,1)$ & $27(10,6)$ \\
\hline & $\mathrm{K}$ & 0,57 & 0,94 & $3(2,1)$ & $9(6,4)$ & $60(42,6)$ & $42(29,8)$ & $27(19,1)$ \\
\hline \multirow{2}{*}{$\begin{array}{l}08 \text { Berufliche Situation für } \\
\text { Bezugsperson }\end{array}$} & $E$ & 0,25 & 0,76 & $3(1,2)$ & $21(8,3)$ & $159(62,6)$ & $52(20,5)$ & $19(7,5)$ \\
\hline & $\mathrm{K}$ & - & - & - & - & - & - & - \\
\hline \multirow[t]{2}{*}{09 Familiäre Situation } & $E$ & 0,17 & 0,75 & $5(2,0)$ & $27(10,6)$ & $154(60,6)$ & $57(22,4)$ & $11(4,3)$ \\
\hline & $\mathrm{K}$ & - & - & - & - & - & - & - \\
\hline \multirow{2}{*}{$\begin{array}{l}10 \text { Veränderungen für Kind } \\
\text { insgesamt }\end{array}$} & $\mathrm{E}$ & 0,83 & 0,95 & $3(1,2)$ & $27(10,6)$ & $41(16,1)$ & $123(48,4)$ & $60(23,6)$ \\
\hline & $\mathrm{K}$ & 0,71 & 0,96 & $3(2,1)$ & $14(9,9)$ & $31(22,0)$ & $66(46,8)$ & $27(19,1)$ \\
\hline \multirow{2}{*}{$\begin{array}{l}11 \text { Veränderungen für } \\
\text { Bezugsperson insgesamt }\end{array}$} & $\mathrm{E}$ & 1,02 & 0,85 & $4(1,6)$ & $10(3,9)$ & $35(13,8)$ & $134(52,8)$ & $71(28,0)$ \\
\hline & $\mathrm{K}$ & - & - & - & - & - & - & - \\
\hline \multirow[t]{3}{*}{ CBB-Gesamtbelastungsindex } & $E$ & 0,55 & 0,52 & & & & & \\
\hline & $E(C)$ & 0,60 & 0,60 & & & & & \\
\hline & $\mathrm{K}$ & 0,61 & 0,54 & & & & & \\
\hline
\end{tabular}

während der Pandemie weder im Eltern- $(F(3,250)=1,61, p=0,19)$ noch im Selbsturteil $(F(3,137)=1,37, p=0,26)$. Allerdings lassen sich geringe, aber statistisch signifikante Zusammenhänge zwischen der von den Eltern bzw. den Kindern und Jugendlichen beurteilten psychischen Belastung und emotionalen und Verhaltensauffälligkeiten insgesamt nachweisen (CBCL-Gesamtauffälligkeit: $r=0,20, \quad p<0,05$; YSR-Gesamtauffälligkeit: $r=0,25, p<0,01)$. Die Zusammenhänge lassen sich ebenso für viele Subskalen des CBCL bzw. YSR belegen (s. Onlinematerial 6). Auch zwischen Belastung und Funktionsbeeinträchtigungen, einschließlich subjektiven Leidensdrucks, im Eltern- bzw. Selbsturteil lassen sich signifikante Zusammenhänge finden (FBB-SCREEN: $r=0,13, p<0,05$; SBB-SCREEN: $r=0,36, p<0,01)$.
Der Anteil der Kinder und Jugendlichen mit emotionalen und Verhaltensauffälligkeiten in der(Vor-)Schulstichprobe liegt bei Anwendung der vorgegeben Cut-offs mit 11,4\% (Elternurteil) bzw. 10,0\% (Selbsturteil) im Bereich der durch die Normierungen dieser Instrumente erwarteten Auffälligkeitsraten [26, 27]. Auch in der (Vor-)Schulstichprobe lassen sich ähnlich hohe Zusammenhänge zwischen der von den Eltern bzw. den Kindern und Jugendlichen beurteilten psychischen Belastung und emotionalen und Verhaltensauffälligkeiten insgesamt nachweisen (SDQ-E Gesamtauffälligkeit: $r=0,33, p<0,01$; SDQ-S Gesamtauffälligkeit: $r=0,22, p<0,01)$. Die Zusammenhänge lassen sich auch für fast alle Subskalen des SDQ-E bzw. SDQ-S belegen.

\section{Diskussion}

Die vorliegende multizentrische Analyse untersucht das Ausmaß der psychischen Belastung von Kindern und Jugendlichen und ihren Familien während der COVID-19-Pandemie und den Zusammenhang mit emotionalen und Verhaltensauffälligkeiten, mit negativ erlebten Veränderungen im Umfeld sowie mit soziodemografischen Parametern. Dazu wurden die Daten aus 2 Stichproben parallel analysiert: eine klinische Inanspruchnahmestichprobe aus kinder- und jugendpsychiatrischen sowie kinder- und jugendlichenpsychotherapeutischen Ambulanzen und eine Feldstichprobe von Kindern und Jugendlichen, die vorschulische und schulische Einrichtungen in verschiedenen Städten Deutschlands besuchen. 
Tab. 5 Stärke der Belastungen durch die Coronapandemie in der (Vor-)Schulstichprobe im Elternurteil ( $n=1606)$ und im Selbsturteil (ab 11 Jahren; $n=1024)$, Angaben auf Itemebene

\begin{tabular}{|c|c|c|c|c|c|c|c|c|}
\hline & \multirow[t]{2}{*}{ Urteil } & \multirow[t]{2}{*}{$M$} & \multirow[t]{2}{*}{$S D$} & \multicolumn{5}{|l|}{$n(\%)$} \\
\hline & & & & $\begin{array}{l}\text { Viel } \\
\text { besser }(-2)\end{array}$ & $\begin{array}{l}\text { Etwas } \\
\text { besser }(-1)\end{array}$ & $\begin{array}{l}\text { Unverän- } \\
\text { dert (0) }\end{array}$ & $\begin{array}{l}\text { Etwas schlech- } \\
\text { ter (1) }\end{array}$ & $\begin{array}{l}\text { Viel schlech- } \\
\text { ter (2) }\end{array}$ \\
\hline \multirow{2}{*}{$\begin{array}{l}01 \text { Beziehungen Kind zu } \\
\text { Familienmitgliedern }\end{array}$} & $\mathrm{E}$ & $-0,03$ & 0,65 & $33(2,1)$ & $236(14,7)$ & $1109(69,2)$ & $200(12,5)$ & $25(1,6)$ \\
\hline & K & 0,00 & 0,69 & $26(2,5)$ & $147(14,4)$ & $661(64,8)$ & $171(16,8)$ & $15(1,5)$ \\
\hline \multirow{2}{*}{$\begin{array}{l}02 \text { Beziehungen Kind zu } \\
\text { Freund:innen }\end{array}$} & $\mathrm{E}$ & 0,45 & 0,75 & $13(0,8)$ & $94(5,9)$ & $770(48,0)$ & $610(38,1)$ & $116(7,2)$ \\
\hline & K & 0,36 & 0,91 & $30(2,9)$ & $140(13,7)$ & $364(35,5)$ & $414(40,4)$ & $76(7,4)$ \\
\hline \multirow{2}{*}{$\begin{array}{l}03 \text { Belastungen durch } \\
\text { Schule/Lernen }\end{array}$} & $\mathrm{E}$ & 0,56 & 0,86 & $18(1,1)$ & $138(8,7)$ & $576(36,2)$ & $653(41,1)$ & $204(12,8)$ \\
\hline & K & 0,48 & 0,96 & $29(2,8)$ & $125(12,2)$ & $327(32,0)$ & $415(40,6)$ & $127(12,4)$ \\
\hline \multirow[t]{2}{*}{04 Betreuung des Kindes } & $\mathrm{E}$ & 0,44 & 0,81 & $23(1,4)$ & $79(5,0)$ & $832(52,2)$ & $498(30,7)$ & $117(10,7)$ \\
\hline & K & - & - & - & - & - & - & - \\
\hline \multirow{2}{*}{$\begin{array}{l}05 \text { Freizeit/Beschäftigungs- } \\
\text { möglichkeit }\end{array}$} & $\mathrm{E}$ & 1,50 & 0,72 & $9(0,6)$ & $15(0,9)$ & $118(7,4)$ & $490(30,5)$ & $973(60,6)$ \\
\hline & K & 1,29 & 0,90 & $16(1,6)$ & $37(3,6)$ & $103(10,1)$ & $345(33,7)$ & $523(51,1)$ \\
\hline \multirow{2}{*}{$\begin{array}{l}\text { 06 Gereiztheit/Unruhe/ } \\
\text { Unausgeglichenheit }\end{array}$} & $E$ & 0,49 & 0,75 & $11(0,7)$ & $93(5,8)$ & $720(45,0)$ & $657(41,1)$ & $118(7,4)$ \\
\hline & K & 0,46 & 0,78 & $12(1,2)$ & $77(7,5)$ & $430(42,1)$ & $432(42,3)$ & $71(6,9)$ \\
\hline \multirow{2}{*}{$\begin{array}{l}07 \text { Ängstlichkeit/Unsicherheit/ } \\
\text { Traurigkeit }\end{array}$} & $E$ & 0,35 & 0,65 & $14(0,9)$ & $55(3,4)$ & $950(59,2)$ & $523(32,6)$ & $62(3,9)$ \\
\hline & K & 0,32 & 0,75 & $18(1,8)$ & $61(6,0)$ & $575(56,2)$ & $310(30,3)$ & $59(5,8)$ \\
\hline \multirow{2}{*}{$\begin{array}{l}08 \text { Berufliche Situation für } \\
\text { Bezugsperson }\end{array}$} & $E$ & 0,42 & 0,81 & $29(1,8)$ & $76(4,8)$ & $865(54,1)$ & $460(28,8)$ & $170(10,6)$ \\
\hline & K & - & - & - & - & - & - & - \\
\hline \multirow[t]{2}{*}{09 Familiäre Situation } & $E$ & 0,14 & 0,73 & $34(2,1)$ & $174(10,9)$ & $993(61,9)$ & $343(21,4)$ & $59(3,7)$ \\
\hline & K & - & - & - & - & - & - & - \\
\hline \multirow{2}{*}{$\begin{array}{l}10 \text { Veränderungen für Kind } \\
\text { insgesamt }\end{array}$} & $E$ & 0,91 & 0,72 & $4(0,2)$ & $62(3,9)$ & $283(17,6)$ & $984(61,3)$ & $271(16,9)$ \\
\hline & K & 0,55 & 0,85 & $20(2,0)$ & $104(10,2)$ & $269(26,4)$ & $546(53,5)$ & $81(7,9)$ \\
\hline \multirow{2}{*}{$\begin{array}{l}11 \text { Veränderungen für } \\
\text { Bezugsperson insgesamt }\end{array}$} & $\mathrm{E}$ & 1,04 & 0,78 & $20(1,2)$ & $51(3,2)$ & $192(12,0)$ & $931(58,0)$ & $411(25,6)$ \\
\hline & K & - & - & - & - & - & - & - \\
\hline \multirow[t]{3}{*}{ CBB-Gesamtbelastungsindex } & $E$ & 0,57 & 0,44 & & & & & \\
\hline & $E(C)$ & 0,60 & 0,46 & & & & & \\
\hline & K & 0,50 & 0,48 & & & & & \\
\hline
\end{tabular}

Zur Berücksichtigung verschiedener Beurteilungsperspektiven wurden sowohl Eltern- als auch Selbsturteile von Kindern und Jugendlichen ab 11 Jahren erfasst.

Insgesamt lagen die Belastungsindizes auf einer Skala von -2 (starke Entlastung) bis +2 (starke Belastung) in beiden Stichproben und unter beiden Beurteilungsperspektiven im Mittel zwischen 0,5 und 0,6. Die Unterschiede zwischen den Beurteilenden und auch zwischen beiden Stichproben sind weitgehend $\mathrm{zu}$ vernachlässigen, d.h., sowohl bei einer überwiegend unauffälligen (Vor-)Schulstichprobe als auch bei einer psychisch auffälligen Klinikstichprobe lassen sich im Durchschnitt leichte bis moderate Belastungen im Verlauf der Pandemie aus den Berichten von Eltern und ihren Kindern erkennen. Lediglich im Urteil der Kinder und Jugendlichen konnte in der
Klinikstichprobe eine signifikante und gering bis moderat erhöhte Belastung im Vergleich zur (Vor-)Schulstichprobe festgestellt werden. Aus diesem Befund lässt sich jedoch nicht schlussfolgern, dass bei allen Kindern und Familien nur leichte Belastungen $\mathrm{zu}$ eruieren sind, wie eine detaillierte Analyse der Daten zeigt. Insgesamt beschreiben rund $60-70 \%$ der Eltern und der Kinder und Jugendlichen sowohl in der klinischen als auch in der Feldstichprobe eine Zunahme an Belastungen, wobei eine starke Belastung bei rund 10-25\% der Fälle festzustellen ist. Dieses Ergebnis korrespondiert mit dem Ergebnis der bundesweit repräsentativen Studie von Ravens-Sieberer et al. [12, 13], nach der $71 \%$ der Kinder und Jugendlichen und $75 \%$ der Eltern eine Belastung durch die erste Pandemiewelle angaben. In der vorliegenden Studie kann das Ausmaß der Belastung dahin gehend diffe- renziert werden, dass in der Mehrzahl der Fälle eine geringe bis moderate Belastung berichtet wird.

Darüber hinaus beschreiben rund $12 \%$ der Eltern und der Kinder und Jugendlichen in der Klinikstichprobe eine Entlastung für das Kind/den Jugendlichen während der Pandemie. In der (Vor-)Schulstichprobe empfinden das auch rund $12 \%$ der Kinder und Jugendlichen so, aber nur $4 \%$ der Eltern. Rund $17-24 \%$ der Eltern oder Kinder und Jugendlichen in beiden Stichproben berichten von einer Verbesserung der familiären Beziehungen während der Pandemie. Diese Ergebnisse entsprechen den Ergebnissen von Cost et al. [20], nach denen bei 19-31\% der Kinder und Jugendlichen in mindestens einem Bereich eine Verbesserung der psychischen Gesundheit beschrieben wird. Eine Entlastung durch geringere 
schulische Anforderungen aufgrund der Beschulung im häuslichen Umfeld dürfte allerdings nur passager sein.

Auch hinsichtlich einzelner Belastungsfaktoren lassen sich vergleichbare Ergebnisse bei der (Vor-)Schulstichprobe und der COPSY-Studie belegen: So beschreiben rund $53 \%$ der Kinder und Jugendlichen höhere Belastungen bezüglich Schule und Lernen (COPSY: $64 \%$ ) und $48 \%$ beschreiben eine Verschlechterung der Beziehungen zu ihren Freund:innen (COPSY: 39\%). Diese Ähnlichkeiten sind ein weiterer Hinweis auf die Repräsentativität der Ergebnisse aus der (Vor-)Schulstichprobe. Die mit Abstand stärksten Belastungen sehen sowohl Eltern als auch Kinder und Jugendliche durch die reduzierten Freizeitund Beschäftigungsmöglichkeiten - hier beschreiben rund $70-90 \%$ leichte bis starke Verschlechterungen.

Die psychischen Auffälligkeiten in Form von Gereiztheit, Unruhe und Unausgeglichenheit oder in Form von Ängstlichkeit, Unsicherheit oder Traurigkeit haben sich in der Klinikstichprobe sowohl im Eltern- als auch im Selbsturteil im Vergleich zur Feldstichprobe etwa doppelt so häufig stark verschlechtert. Dies weist hin auf eine erhöhte Vulnerabilität für eine stärkere Zunahme von emotionalen und Verhaltensauffälligkeiten in der Klinikstichprobe bei im Durchschnitt vergleichbar starker, d.h. überwiegend leichter bis moderater Belastungszunahme durch die Pandemie. Dies entspricht auch der Einschätzung der Direktor:innen kinder- und jugendpsychiatrischer Kliniken in Europa [31], die ebenfalls eine moderate Belastung der Patient:innen und eine Zunahme der Inanspruchnahme berichten.

Zwar konnten im Gegensatz zu manchen anderen Studien (z. B. $[12,13])$ keine substanziellen soziodemografischen Faktoren gefunden werden (z.B. Alter, Geschlecht, sozioökonomischer Status), welche die Belastung durch COVID-19 deutlich beeinflussen; allerdings zeigen sich leichte bis moderate Zusammenhänge zwischen der subjektiv erlebten Verschlechterung der familiären und sozialen Situation und einem erhöhten Belastungserleben.
Die COPSY-Studie [13] berichtet von erhöhten psychischen Auffälligkeiten in der ersten Pandemiephase im Elternurteil (17,8\%) im Vergleich zu einer Erhebung vor Ausbruch von COVID19-Pandemie. Unter Verwendung des gleichen Fragebogenverfahrens (SDQElternurteil) konnten in der (Vor-)Schulstichprobe mit $11,4 \%$ allerdings keine so deutlich erhöhten Werte festgestellt werden. Dies mag mit dem Erhebungszeitpunkt (Pandemiewelle 2 bis Mitte Pandemiewelle 3) oder auch mit einer begrenzten Repräsentativität der vorliegenden (Vor-)Schulstichprobe zusammenhängen.

Schließlich konnten auch leichte bis moderate Zusammenhänge zwischen Belastungen und psychischen Auffälligkeiten sowohl im Selbst- als auch im Fremdurteil in beiden Stichproben nachgewiesen werden, wobei die Beeinflussungsrichtungen aus diesen Korrelationen nicht erschlossen werden können: Belastungen können zu stärkeren psychischen Auffälligkeiten beitragen und/oder stärkere psychische Auffälligkeiten können Belastungen erhöhen. Grundsätzlich handelt es sich um Querschnittserhebungen, somit lassen die vorgenommenen Korrelationsanalysen keine Ableitung kausaler Zusammenhänge zu.

Neben den Stärken der Studie - vor allem die parallele Betrachtung von Elternund Selbsteinschätzungen in einer Feldstichprobe und einer Klinikstichprobe sind einige Limitationen $\mathrm{zu}$ beachten. Trotz Bemühungen, Daten konsekutiv und multizentrisch zu erheben, um eine möglichst hohe Repräsentativitätsrate in der klinischen Inanspruchnahmestichprobe zu erreichen, gelang dies aufgrund von hohen "Schwundraten“ und stark unterschiedlichen Teilnahmequoten der einzelnen Standorte nicht. Da eine weitere Fragestellung der Studie sich auf die Durchführung von Ferntherapie bezog, wurden außerdem Patient:innen mit einer Kontraindikation für Videotherapie nicht in die Klinikstichprobe aufgenommen. Dadurch wurden Patient:innen mit stark ausgeprägten psychischen Störungen nicht berücksichtigt. Insofern ist die Repräsentativität der Klinikstichprobe eingeschränkt. Auch die Repräsentativität der Feldstichprobe ist vor allem durch die lokale Verteilung und die unterschiedlich hohen Teilnahmequoten in den Einrichtungen eingeschränkt. Die Studie ist lediglich eine Momentaufnahme während des zweiten Lockdowns, die Aussagen bezüglich subjektiv erlebter Belastungen ermöglicht. Das Ausmaß der objektiven Einschränkungen wurde nicht erhoben und Aussagen über die Stabilität der identifizierten Belastungen können nicht getroffen werden. Außerdem wurden beide Stichproben nicht über statistische Verfahren direkt miteinander verglichen und Unterschiede in der Zusammensetzung beider Stichproben wurden nicht korrigiert. Mit dieser Publikation sollten die basalen Ergebnisse für jede Stichprobe dargestellt werden. Komplexere Analysen sind weiteren Publikationen vorbehalten.

\section{Fazit}

Die Ergebnisse der Studie unterstreichen die Notwendigkeit während einer Pandemie, belastete Subgruppen sowohl in klinischen Stichproben als auch in der Gesamtbevölkerung zu identifizieren und gezielte Interventionen anzubieten. Wegen des hohen Anteils von Kindern und Jugendlichen ohne ausgeprägte $\mathrm{Be}$ lastung sind universelle Interventionen nicht indiziert.

\section{Korrespondenzadresse}

Prof. Dr. Dipl.-Psych. Manfred Döpfner Klinik für Psychiatrie, Psychosomatik und Psychotherapie des Kindes- und Jugendalters, Medizinische Fakultät und Uniklinik Köln, Universität zu Köln

Robert-Koch-Str. 10, 50931 Köln, Deutschland manfred.doepfner@uk-koeln.de

Mitglieder der TEMPO-Studiengruppe. Manfred Döpfner (Ausbildungsinstitut für Kinder- und Jugendlichenpsychotherapie (AKiP), Medizinische Fakultät und Uniklinik Köln, Universität zu Köln, Köln, Deutschland; Klinik für Psychiatrie, Psychosomatik und Psychotherapie des Kindes- und Jugendalters, Medizinische Fakultät und Uniklinik Köln, Universität zu Köln, Köln, Deutschland); Julia Adam (Ausbildungsinstitut für Kinder- und Jugendlichenpsychotherapie (AKiP), Medizinische Fakultät und Uniklinik Köln, Universität zu Köln, Köln, Deutschland); Carolina Habbel (Ausbildungsinstitut für Kinder- und Jugendlichenpsychotherapie (AKiP), Medizinische Fakultät und Uniklinik Köln, Universität zu Köln, Köln, Deutschland); Karen Schulze-Husmann (Ausbildungsinstitut für Kinder- und Jugendlichenpsychotherapie (AKiP), Medizinische Fakultät und Uniklinik 
Köln, Universität zu Köln, Köln, Deutschland); Michael Simons (Klinik für Psychiatrie, Psychosomatik und Psychotherapie des Kindes- und Jugendalters, RWTH Aachen, Aachen, Deutschland); Jan Schwendowius (Klinik für Psychiatrie, Psychosomatik und Psychotherapie des Kindes- und Jugendalters, Charité, Universitätsmedizin Berlin, Berlin, Deutschland); Fabiola Heuer (Klinik für Psychiatrie, Psychosomatik und Psychotherapie des Kindes- und Jugendalters, Charité, Universitätsmedizin Berlin, Berlin, Deutschland); Christiane Wegner (Klinik für Kinder- und Jugendpsychiatrie/Psychotherapie, Universitätsmedizin Göttingen, Georg-August-Universität Göttingen, Göttingen, Deutschland); Stephan Bender (Klinik für Psychiatrie, Psychosomatik und Psychotherapie des Kindes- und Jugendalters, Medizinische Fakultät und Uniklinik Köln, Universität zu Köln, Köln, Deutschland); Beate Herpertz-Dahlmann (Klinik für Psychiatrie, Psychosomatik und Psychotherapie des Kindes- und Jugendalters, RWTH Aachen, Aachen, Deutschland); Luise Poustka (Klinik für Kinderund Jugendpsychiatrie/Psychotherapie, Universitätsmedizin Göttingen, Georg-August-Universität Göttingen, Göttingen, Deutschland)

Mitglieder der B-FAST-Studiengruppe. Birte Schulte (Klinik für Psychiatrie, Psychosomatik und Psychotherapie des Kindes- und Jugendalters, Medizinische Fakultät und Uniklinik Köln, Universität zu Köln, Köln, Deutschland); Stephan Bender (Klinik für Psychiatrie, Psychosomatik und Psychotherapie des Kindes- und Jugendalters, Medizinische Fakultät und Uniklinik Köln, Universität zu Köln, Köln, Deutschland); Juliane Münch (Klinik für Allgemeine Pädiatrie, Neonatologie und Kinderkardiologie, Universitätsklinikum Düsseldorf, Düsseldorf, Deutschland); Burkhard Tönshoff (Klinik Kinderheilkunde I, Zentrum für Kinder- und Jugendmedizin, Universitätsklinikum Heidelberg, Heidelberg, Deutschland); Alexander Joachim (Klinik für Kinder- und Jugendmedizin, Medizinische Fakultät und Uniklinik Köln, Universität zu Köln, Köln, Deutschland); Lena T. Birzele (Kinderklinik und Kinderpoliklinik, LMU Klinikum der Universität München, München, Deutschland); Eva Möhler (Klinik für Kinder- und Jugendpsychiatrie, Psychosomatik und Psychotherapie, Universitätsklinik Saarland und Medizinische Fakultät der Universität des Saarlandes, Homburg, Deutschland)

Förderung. Die Studie wurde im Rahmen des „egePan Unimed - Entwicklung, Testung und Implementierung von regional adaptiven Versorgungsstrukturen und Prozessen für ein evidenzgeleitetes Pandemiemanagement " durchgeführt. egePan Unimed wird im Rahmen des Netzwerks Universitätsmedizin (NUM) gefördert vom Bundesministerium für Bildung und Forschung (BMBF; Förderkennzeichen: 01KX2021) und steht unter der Gesamtprojektleitung von Prof. Dr. Jochen Schmitt und Dr. Michael von Wagner.

Funding. Open Access funding enabled and organized by Projekt DEAL.

\section{Einhaltung ethischer Richtlinien}

Interessenkonflikt. J. Adam, C. Habbel, B. Schulte, K. Schulze-Husmann, M. Simons, F. Heuer und C. Wegner geben an, dass kein Interessenkonflikt besteht. M. Döpfner ist Autor von Diagnostikinstrumenten, die im Rahmen der Studie Verwendung fanden und für welche er Honorare vom Hogrefe Verlag erhält. S. Bender hat eine öffentliche Drittmittelförderung (NUM Netzwerk Universitätsmedizin) zu dem im Rahmen der Studie untersuchten Themengebiet erhalten.

Die Umsetzung der TEMPO-Studie wurde von der Ethikkommission der Medizinischen Fakultät der Universität zu Köln (Zeichen 20-1537) sowie darauffolgend von allen weiteren beteiligten Studienzentren zustimmend bewertet, Gleiches gilt für die B-FASTStudie (Zeichen 20-1463). Alle beschriebenen Befragungen wurden im Einklang mit nationalem Recht sowie gemäß der Deklaration von Helsinki von 1975 (in der aktuellen, überarbeiteten Fassung) durchgeführt. Von allen beteiligten Patient:innen und Proband:innen liegt eine Einverständniserklärung vor.

Open Access. Dieser Artikel wird unter der Creative Commons Namensnennung 4.0 International Lizenz veröffentlicht, welche die Nutzung, Vervielfältigung, Bearbeitung, Verbreitung und Wiedergabe in jeglichem Medium und Format erlaubt, sofern Sie den/die ursprünglichen Autor(en) und die Quelle ordnungsgemäß nennen, einen Link zur Creative Commons Lizenz beifügen und angeben, ob Änderungen vorgenommen wurden.

Die in diesem Artikel enthaltenen Bilder und sonstiges Drittmaterial unterliegen ebenfalls der genannten Creative Commons Lizenz, sofern sich aus der Abbildungslegende nichts anderes ergibt. Sofern das betreffende Material nicht unter der genannten Creative Commons Lizenz steht und die betreffende Handlung nicht nach gesetzlichen Vorschriften erlaubt ist, ist für die oben aufgeführten Weiterverwendungen des Materials die Einwilligung des jeweiligen Rechteinhabers einzuholen.

Weitere Details zur Lizenz entnehmen Sie bitte der Lizenzinformation auf http://creativecommons.org/ licenses/by/4.0/deed.de.

\section{Literatur}

1. Cluver L, Lachman JM, Sherr L, Wessels I, Krug E, Rakotomalala S, McDonald K (2020) Parenting in a time of COVID-19. Lancet 395(10231):e64. https://doi.org/10.1016/S0140-6736(20)30736-4

2. Fegert JM, Vitiello B, Plener PL, Clemens V (2020) Challenges and burden of the Coronavirus 2019 (COVID-19) pandemic for child and adolescent mental health: a narrative review to highlight clinical and research needs in the acute phase and the long return to normality. Child Adolesc Psychiatry Ment Health 14:20. https://doi.org/10. 1186/s13034-020-00329-3

3. Paschke K, Arnaud N, Austermann MI, Thomasius R (2021) Risk factors for prospective increase in psychological stress during COVID-19 lockdown in a representative sample of adolescents and their parents. BJPsych open 7(3):e94. https://doi.org/10. 1192/bjo.2021.49

4. Ravens-Sieberer U, Kaman A, Erhart M, Otto C, Devine J, Löffler C, Hurrelmann K, Hölling H (2021) Quality of life and mental health in children and adolescents during the first year of the COVID19 pandemic in Germany: results of a two-wave nationally representative study. SSRN. https://doi org/10.2139/ssrn.3798710

5. Tang S, Xiang M, Cheung T, Xiang YT (2021) Mental health and its correlates among children and adolescents during COVID-19 school closure: the importance of parent-child discussion. J Affect
Disord 279:353-360. https://doi.org/10.1016/j. jad.2020.10.016

6. Calvano C, Engelke L, Di Bella J, Kindermann J, Renneberg B, Winter SM (2021) Families in the COVID-19 pandemic: parental stress, parent mental health and the occurrence of adverse childhood experiences-results of a representative survey in Germany. Eur Child Adolesc Psychiatry. https://doi.org/10.1007/s00787-021-01739-0

7. Paschke K, Austermann Ml, Simon-Kutscher K, Thomasius R (2021) Adolescent gaming and social media usage before and during the COVID-19 pandemic. Sucht 67:13-22. https://doi.org/10. 1024/0939-5911/a000694

8. Brown SM, Doom JR, Lechuga-Pena S, Watamura S, Koppels T (2020) Stress and parenting during the global COVID-19 pandemic. Child Abuse Negl. https://doi.org/10.1016/j.chiabu.2020.104699

9. Duan L, Shao $X$, Wang $Y$, Huang $Y$, Miao J, Yang X, Zhu G (2020) An investigation of mental health status of children and adolescents in china during the outbreak of COVID-19. J Affect Disord 275:112-118. https://doi.org/10.1016/j.jad.2020. 06.029

10. Xie X, Xue Q, Zhou Y, Zhu K, Liu Q, Zhang J, Song R (2020) Mental health status among children in home confinement during the Coronavirus disease 2019 Outbreak in Hubei Province, China. JAMA Pediatr 174(9):898-900. https://doi.org/10.1001/ jamapediatrics.2020.1619

11. Breaux R, Dvorsky MR, Marsh NP, Green CD, Cash AR, Shroff DM, Becker SP (2021) Prospective impact of COVID-19 on mental health functioning in adolescents with and without ADHD: protective role of emotion regulation abilities. J Child Psychol Psychiatry.https://doi.org/10.1111/jcpp.13382

12. Ravens-Sieberer U, Kaman A, Otto C, Adedeji A, Napp AK, Becker M, Hurrelmann K (2021) Seelische Gesundheit und psychische Belastungen von Kindern und Jugendlichen in der ersten Welle der COVID-19-Pandemie - Ergebnisse der COPSYStudie. Bundesgesundheitsblatt. https://doi.org/ 10.1007/s00103-021-03291-3

13. Ravens-Sieberer U, Kaman A, Erhart M, Devine J, Schlack R, Otto C (2021) Impact of the COVID19 pandemic on quality of life and mental health in children and adolescents in Germany. Eur Child Adolesc Psychiatry. https://doi.org/10.1007/ s00787-021-01726-5

14. Tso WWY, Wong RS, Tung KTS, Rao N, Fu KW, Yam JCS, Lp P (2020) Vulnerability and resilience in children during the COVID-19 pandemic. Eur Child Adolesc Psychiatry. https://doi.org/10.1007/ s00787-020-01680-8

15. Thorell LB, Skoglund C, de la Pena AG, Baeyens D, Fuermaier ABM, Groom MJ, Christiansen H (2021) Parental experiences of homeschooling during the COVID-19 pandemic: differences between seven European countries and between children with and without mental health conditions. Eur Child Adolesc Psychiatry. https://doi.org/10.1007/ s00787-020-01706-1

16. Bobo E, Lin L, Acquaviva E, Caci H, FrancN, Gamon L, Purper-Ouakil D (2020) How do children and adolescents with Attention Deficit Hyperactivity Disorder (ADHD) experience lockdown during the COVID-19 outbreak? Encephale 46(3S):85-S92. https://doi.org/10.1016/j.encep.2020.05.011

17. Zhang J, Shuai L, Yu H, Wang Z, Qiu M, Lu L, Chen R (2020) Acute stress, behavioural symptoms and mood states among school-age children with attention-deficit/hyperactive disorder during the COVID-19 outbreak. Asian J Psychiatry. https://doi. org/10.1016/j.ajp.2020.102077 
18. Ivanova MY, Achenbach TM, Rescorla LA, Guo J, Althoff RR, Kan K-J, Verhulst FC (2019) Testing syndromes of psychopathology in parent and youth ratings across societies. J Clin Child Adolesc Psychol 48:596-609. https://doi.org/10.1080/ 15374416.2017.1405352

19. Plück J, Döpfner M, Berner W, Fegert J, Huss $M$, Lenz K, Schmeck K, Lehmkuhl U, Poustka F, Lehmkuhl G (1997) Die Bedeutung unterschiedlicher Informationsquellen bei der Beurteilung psychischer Störungen im Jugendalter - ein Vergleich von Elternurteil und Selbsteinschätzung der Jugendlichen. Prax Kinderpsychol Kinderpsychiatr 46:566-582

20. Cost KT, Crosbie J, Anagnostou E, Birken CS, Charach A, Monga S, Korczak DJ (2021) Mostly worse, occasionally better: impact of COVID-19 pandemic on the mental health of Canadian children and adolescents. Eur Child Adolesc Psychiatry. https://doi.org/10.1007/s00787-02101744-3

21. Dvorsky MR, Breaux R, Becker SP (2020) Finding ordinary magic in extraordinary times: child and adolescent resilience during the COVID-19 pandemic. Eur Child Adolesc Psychiatry 1:1-3. https://doi.org/10.1007/s00787-020-01583-8

22. Bruining $\mathrm{H}$, Bartels $M$, Polderman TJ, Popma $A$ (2020) COVID-19 and child and adolescent psychiatry: an unexpected blessing for part of our population? Eur Child Adolesc Psychiatry. https:// doi.org/10.1007/s00787-020-01578-5

23. Döpfner M, Görtz-Dorten A (2020). Corona Belastungsbogen. Unveröffentlicht, Klinik für Psychiatrie, Psychosomatik und Psychotherapie des Kindes- und Jugendalters an der Uniklinik Köln

24. Döpfner M, Plück J, Kinnen C, Arbeitsgruppe Deutsche Child Behavior Checklist (2014) Deutsche Schulalter-Formen der Child Behavior Checklist von Thomas M. Achenbach. Hogrefe, Göttingen (Elternfragebogen über das Verhalten von Kindern und Jugendlichen, $(\mathrm{CBCL} / 6-18 \mathrm{R})$, Lehrerfragebogen über das Verhalten von Kindern und Jugendlichen (TRF/6-18R), Fragebogen für Jugendliche (YSR/11-18R))

25. Goodman R (1997) The Strengths and Difficulties Questionnaire. A research note. J Child Psychol Psychiatry 38:581-586

26. Woerner W, Becker A, Friedrich C, Rothenberger A, Klasen H, Goodman R (2002) Normierung und Evaluation der deutschen Elternversion des Strengths and Difficulties Questionnaire (SDQ): Ergebnisse einer repräsentativen Felderhebung. Z Kinder Jugendpsychiatr Psychother 30:105-112. https://doi.org/10.1024//1422-4917.30.2.105

27. LohbeckA, SchultheißJ, Petermann F, Petermann U (2015) Die deutsche Selbstbeurteilungsversion des Strengths and DifficultiesQuestionnaire (SDQ-Deu-S) Psychometrische Eigenschaften, Faktorenstruktur und Grenzwerte. Diagnostica 61:222-235. https://doi.org/10.1026/0012-1924/ a000153

28. Döpfner M, Görtz-Dorten A (2017) DiagnostikSystem für psychische Störungen nach ICD-10 und DSM-5 für Kinder- und Jugendliche (DISYPS-III). Hogrefe, Bern

29. Remschmidt H, Schmidt MH, Poustka F (2006) Multiaxiales Klassifikationsschema für psychische Störungen des Kindes- und Jugendalters nach ICD10 derWHO, 5. Aufl. Huber, Bern

30. Döpfner M, Steinhausen H-C (2012) Störungsübergreifende Verfahren zur Diagnostik psychischer Störungen. Kinder-Diagnostik-System (KIDS), Bd. 3. Hogrefe, Göttingen
31. Revet A, Hebebrand J, Anagnostopoulos D, Kehoe LA, COVID-19 Child and Adolescent Psychiatry Consortium, Kehoe LA (2021) ESCAP CovCAP survey of heads of academic departments to assess the perceived initial (April/May 2020) impact of the COVID-19 pandemic on child and adolescent psychiatry services. Eur Child Adolesc Psychiatr. https://doi.org/10.1007/s00787-02001699-x 